\title{
Visual analysis of geospatial multivariate data for investigating radioactive deposition processes
}

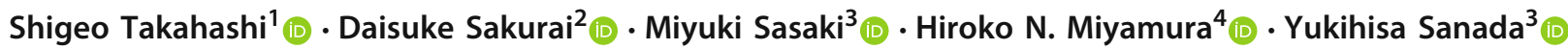

Accepted: 6 July 2021 / Published online: 20 July 2021

(C) The Author(s) 2021

\begin{abstract}
The Fukushima nuclear accident of 2011 raised awareness of the importance of radioactive deposition processes, especially for proposing aerosol measures against possible air pollution. However, identifying these types of processes is often difficult due to complicated terrains. This paper presents an application study for identifying radioactive deposition processes by taking advantage of visual interaction with topographic data. The idea is to visually investigate the correspondence of the spatial positions to the air dose rate along with relevant attributes. This is accomplished by composing scatterplots of pairwise attributes, onto which we project terrain areas to interactively find specific patterns of such attributes. We applied our approach to the analysis of air dose rate distribution data around the Fukushima nuclear plant after the accident. Our visualization technique clearly distinguished contamination areas derived from different deposition processes and thus is useful for elucidation of the deposition process.
\end{abstract}

Keywords Fukushima Daiichi nuclear power plant accident · Topographic analysis · Deposition processes · Continuous scatterplots

\section{Introduction}

It has been more than 10 years since the Fukushima Daiichi Nuclear Power Plant (FDNPP) accident, which was

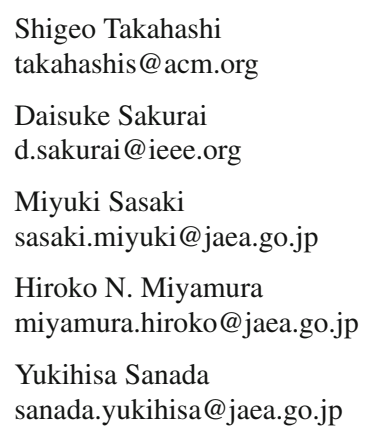

Shigeo Takahashi

takahashis@acm.org

Daisuke Sakurai

d.sakurai@ieee.org

Miyuki Sasaki

sasaki.miyuki@jaea.go.jp

Hiroko N. Miyamura

miyamura.hiroko@jaea.go.jp

Yukihisa Sanada

sanada.yukihisa@jaea.go.jp

1 Department of Computer Science and Engineering, University of Aizu, Aizu-Wakamatsu 965-8580, Japan

2 Pan-Omics Data Driven Innovation Research Center at the Research Institute for Information Technology, Kyushu University, Kasuga 816-8580, Japan

3 Sector of Fukushima Research and Development, Japan Atomic Energy Agency, Minami-soma 975-0036, Japan

4 Center for Computational Science and E-systems and Nuclear Human Resource Development Center, Japan Atomic Energy Agency, Tokyo 100-0011, Japan caused by the March 2011 East Japan earthquake and ensuing tsunami. The disaster caused meltdowns in several units of the FDNPP and the spread of radioactive materials over the surrounding areas. Ever since, ongoing soil decontamination work has sought to reduce the radioactive material to a safe level. The associated restricted residential region, which is located approximately $10 \mathrm{~km}$ away, has shrunk considerably as a result. Additionally, entry into the $10-\mathrm{km}$ zone of the FDNPP remains restricted. This dedicated recovery from the earthquake's damage has allowed us to conduct a more intensive post-analysis of the spatiotemporal behavior of the ambient air dose rate. In particular, understanding the process of radioactive deposition is important because the air dose rate is not proportional to the distance from the FDNPP but rather depends on the deposition process. This is also useful not only for systematic future planning for environmental restoration but also for effective measures against air pollution caused by other particulate materials, such as PM2.5. More extensive investigations into the process are ongoing, with the Japanese government collecting data on the spatiotemporal distribution of the air dose rate through ground and airborne radiation monitoring [20,22].

To understand radioactive deposition processes, it is important to classify them into several categories in terms 
Fig. 1 Interactive analysis of relationships between the geospatial domain and relevant topographic attributes.

Meaningful correspondences between the domain and range are identified by painting projected data samples in the three scatterplots of the pairwise attribute values (a) and those in the map domain (b). The three function values $P, Q$, and $R$ represent the air dose rate, altitude from the ground, and distance from the FDNPP, respectively. a Three scatterplots in the $2 \mathrm{D}$ ranges spanned by $P-Q, P-R$, and $Q-R$, in descending order. b Map domain around the FDNPP. c Interface for interactive analysis

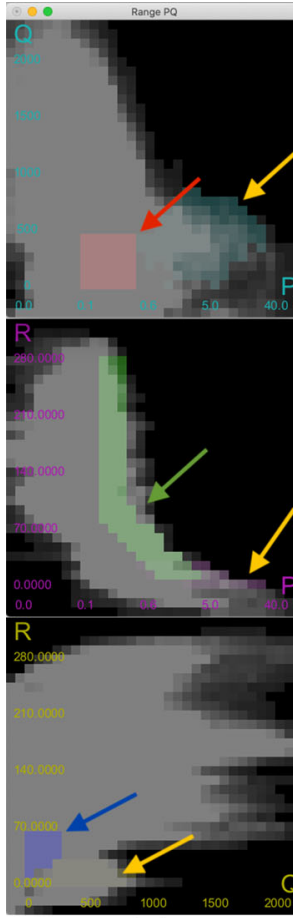

(a)

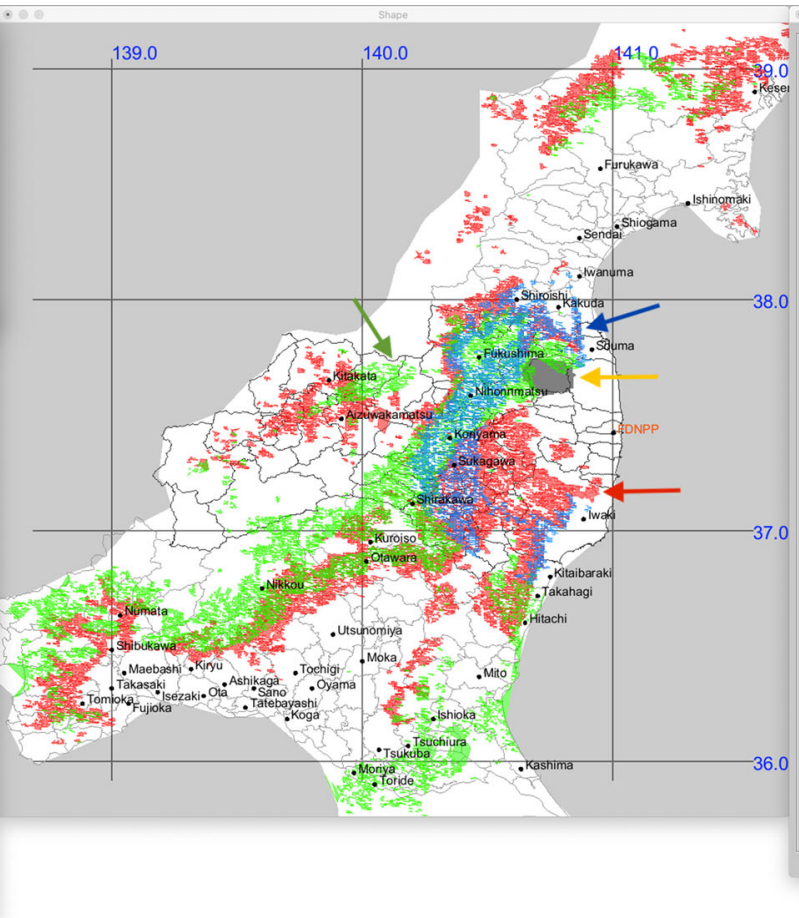

(b)

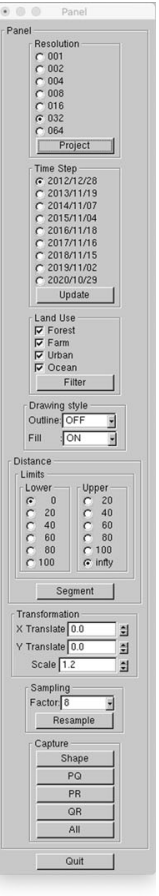

(c) of decomposition mechanisms and land-use patterns. With this information, we can properly identify how to reduce the radioactive level in the corresponding area through decontamination work, including soil cleaning and disposal. Such identification is usually carried out by simulating the spatiotemporal behavior of wind just after the accident as well as analyzing measured rainfall data for the area [19]. However, precisely identifying the type of radioactive deposition process in a specific area is a time-consuming task since it requires inferring a correct model of the deposition mechanism from the available data. This can be further complicated when the target land surface has complex terrain shapes, including steep mountains and valleys. The current scientific knowledge about the deposition process reveals several meaningful relationships between deposition types, land surface shapes, and the associated use patterns in the area. This knowledge can be used to identify the types of deposition processes in the areas around the FDNPP through visual analysis of the spatial distributions of the air dose rate and relevant topographic features.

In this paper, we present an application study for investigating radioactive deposition processes by taking advantage of visual interaction with the topographic features around the FDNPP. The idea was to visually identify the correspondence of the spatial positions to the specific air dose rate together with relevant attributes, including the altitude from the ground, distance from the FDNPP, and degree of attenuation in the air dose rate over time. This was accom- plished by composing scatterplots of pairwise attributes, onto which we projected terrain areas and interacted with them to find specific patterns in these attributes. We tested our approach using the air dose rate distribution data around the FDNPP after the Great East Japan Earthquake disaster of 2011.

Figure 1 presents a snapshot of our visualization system for interactively identifying relationships between the geospatial domain and the range spanned by the attribute values. Figure 1a shows three scatterplot-like charts obtained by projecting the 3D range with respect to each pair of attribute values. Here, $P, Q$, and $R$ indicate the air dose rate, altitude from the ground, and distance from the FDNPP, respectively. By drawing specific patterns of the ranges on the charts, we extracted local areas with specific types of deposition processes in the original map domain, as shown in Fig. 1b. Here, the colored areas in the three scatterplots indicated by red, green, and blue arrows (Fig. 1a) correspond to local map areas (Fig. 1b), where specific types of deposition processes can be identified using the interface (Fig. 1c).

The remainder of this paper is organized as follows. Section 2 provides a survey on previous work related to our study. Section 3 describes the visual analysis approach used to interactively identify radioactive deposition types from the distributions of the air dose rate and other relevant topographic features. Section 4 explains the details of the data acquisition and characteristic relationships between the air dose rate and other topographic features obtained through 
the statistical data analysis. Section 5 presents the results of our visual analysis on the radioactive deposition processes in the area around the FDNPP and discusses how the results match up with the investigation conducted by domain experts. Section 6 concludes the paper and refers to possible future extensions.

\section{Related work}

We divided our survey into two topics: geospatial visualization and multivariate data analysis.

\subsection{Geospatial visualization}

Pioneer studies on geospatial visualization successfully advanced traditional map representations to explore multiple attribute values and locate events of multiple types over the regions of interest. Such visual analysis of geospatial multivariate data has been technically challenging, and extensive investigation in this field is still needed. For example, Sopan et al. [24] conducted health care data analyses in which they visualized the associated geospatial multivariate data. Turkay et al. [29] developed an interactive scheme for evaluating the degree of dependency between the attributes and geographical locations through graphical charts called attribute signatures. In the context of geospatial visualization, analyzing spatiotemporal data is usually necessary. Andrienko et al. [1] applied self-organizing maps to the analysis of spatiotemporal data by projecting both the temporal and spatial continuity. Maciejewski et al. [15] composed a set of tools for finding hotspots in both space and time and then understanding proper reasoning for their emergence.

Techniques for visualizing trajectories on maps have also been intensively studied to analyze diversity suggested by multiple possible routes [14] and find trajectories that meet the given filtration criteria [12]. Another important challenge is analyzing a set of events, such as accidents and crimes, on a map. Beecham et al. [3] presented a design framework that visualizes small multiples viewed simultaneously from various perspectives. Meulemans et al. [16] employed the 2D grid alignment of such small multiples while allowing gaps among them to reflect underlying spatial configurations.

Interaction with geospatial data is another factor that must be carefully designed and evaluated, and a variety of interactive lens techniques have been successfully incorporated in this context [27]. Butkiewicz et al. [4] presented a probebased model for interactively investigating multiple local regions in the context of geospatial visualization, which facilitates the selection of regions of interest for further investigation and comparison.

\subsection{Multivariate data analysis}

One challenge in multivariate data analysis is finding patterns that will help us categorize radioactive deposition types by analyzing multivariate data on the map. The data analyst must efficiently find the correlation between the deposition and topography. Widely used techniques for this include scatterplot matrices and parallel coordinate plots. The data analyst considers a multitude of aspects, and important dimensions are then chosen. Although this study employed domain knowledge to pick the relevant variables, previous research has done so with less a priori knowledge. One important lesson is that the process of investigating dimensions should happen together with the analysis of data values themselves [28]. Other challenges include searching for important data subspaces by introducing similarity measures [25], supportive interactions with visual analysis [33], glyph-based scatterplot matrices [32], and simultaneous clustering of data samples and dimensions [30].

One particular problem for analyzing geographical data in a scatterplot is the interpolation [2]. $m$ values distributed over the earth's surface form a field $\boldsymbol{R}^{2} \rightarrow \boldsymbol{R}^{m}$. Pointbased measurements for deposition, however, are intrinsically discrete. Perhaps the most popular is a scatterplot of sampled points. However, this is prone to sampling biases and artifacts [2] —after all, a finite number of sampling points can be barely interpreted as a $2 \mathrm{D}$ continuum. A common solution is kernel density estimation (KDE). The problem with KDE in geographical applications is that it ignores the topology of the points in the field. Sophisticated studies therefore tend to use kriging instead. Though powerful, kriging requires time-consuming consideration of the parameters based on domain expertise and experiments.

Continuous scatterplots [2], which we employ, are a parameter-free alternative to $\mathrm{KDE}$ and kriging for fields over a continuous domain (such as geographical data). A continuous scatterplot calculates the pixel intensities of the scatterplot by approximating the area that maps to the pixel from the terrain surface. The area is computed by interpolating the sampled, and thus discrete, field. A continuous scatterplot is therefore parameter-free once a reasonable tessellation is agreed upon. Continuous scatterplots have also inspired a few research directions, especially regarding topological analysis for visualization [13]. The singularity visible in continuous scatterplots consists of singular fibers [18] and singular points in the singularity theory (i.e., the Jacobi set [9]).

As the singularity tends to be noisy, a level-of-detail control is needed $[7,26]$. For this, the persistent homology of the Reeb graph can be used to extend the Reeb space [10]. The Reeb space is itself an extension of the Reeb graph, 


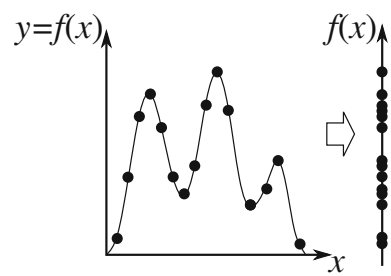

(a)

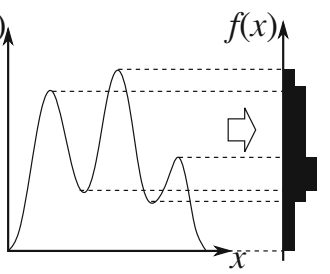

(b)
Fig. 2 1D scatterplots of the function $y=f(x)$. a An ordinary 1D (discrete) scatterplot. b A continuous scatterplot by projecting the continuous representation of the function

which is defined on a single function for multiple functions. Joint contour nets $[5,8]$ have been proposed as a quantized approximation, while a newer algorithm without quantization is now known [26]. This line of research has previously been explored to create a mathematical visualization system [18], establish brush and linking [6], accelerate computation [11], etc.

\section{Analyzing multivariate geospatial data}

We analyzed the mapping from a 2D map domain showing the longitude and latitude of the air dose rate together with a set of attribute values representing the underlying topographic features. For example, we included the altitude from the ground and distance from the FDNPP in the set of attributes. Our challenge was to visualize a set of discrete samples on the multivariate function $f: \boldsymbol{R}^{2} \rightarrow \boldsymbol{R}^{m}$. We incorporated scattered (i.e., integer) values or text labels in addition to continuous ones to classify the map samples into relevant categories. This further improved our understanding of deposition processes by allowing us to filter the data samples according to the expected conditions.

\subsection{Visualizing mappings from 2D map domains}

In this study, we employed continuous scatterplots as our primary tool for the visual analysis of the distribution of multivariate data over the 2D map domain. This tool allowed us to project the distribution of the function in terms of geographical positions, such as the longitude and latitude onto the range space spanned by the air dose rate and other attribute values. Let us begin our explanation with a 1D function $y=f(x)$ as depicted in Fig. 2. By representing the function as a set of discrete samples, we obtained an ordinary 1D scatterplot as the projection of the discrete samples onto the function value $f(x)$ (Fig. 2a). However, by reproducing the continuous form of the function through properly interpolating the discrete samples over the domain, we revealed a more meaningful distribution of the data samples as a 1D continuous scatterplot via projection onto the function value (Fig. 2b).

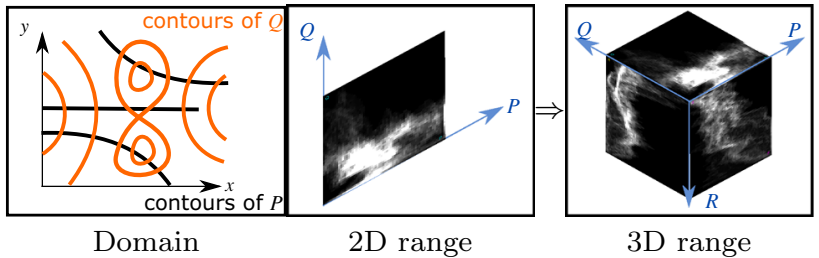

Fig. 3 Pairs of attribute values were selected for investigating the mapping between the geospatial domain and range space

This representation naturally conveys the underlying density map of the data samples and is thus more informative because it reveals the important patterns in the data distribution.

We regarded the continuous scatterplots as the projections of the inverse image of the function $f(x)$, where the mathematical term for the inverse image is a fiber [17]. This effectively allowed us to explore how the regions having specific attribute values behave by tracking the inverse image of the corresponding multivariate function values on the map. Even though we did not necessarily have to track the topological bifurcations of the fibers as has been done in more sophisticated representations, such as joint contour nets $[5,8,26]$, we employed similar computational techniques to obtain the continuous scatterplots. In practice, we employed the method used for visualizing a function $f: \boldsymbol{R}^{3} \rightarrow \boldsymbol{R}^{2}$ in [18], but we reduced the dimensionality of the data domain to $\boldsymbol{R}^{2}$. Moreover, the projected bivariate function space was approximated as a triangulated surface to utilize algorithms for computing joint contour nets [5].

The main technical problem was that we needed an interface to understand the data distribution in the range space spanned by three or more attribute values. Interacting with data samples directly in 3D space usually requires special efforts because we need to recognize its spatial information through our 2D retinal images. Our approach alleviated this problem by coupling two attribute values and projecting the corresponding function onto a $2 \mathrm{D}$ range space individually. This means that we replaced the original 3D range space with multiple $2 \mathrm{D}$ projections by taking advantage of a scatterplot matrix metaphor (Fig. 3). This also helped us better understand the specific patterns inherent in the radioactive deposition processes through ongoing scientific research because such patterns are often characterized by the meaningful relationship between the air dose rate and another attribute value.

\subsection{Interactive exploration}

Our interaction primarily consisted of an interface for investigating the relationship between the geospatial domain and multivariate range space. In practice, scientific modeling of the radioactive deposition process, further described in Sect. 4, allowed us to identify specific patterns in the 
range space spanned by the air dose rate and other topographic attributes. Thus, painting the respective continuous scatterplots effectively facilitated exploration of the correspondence between meaningful patterns in the range space and regions of interest in the map domain. The concept of fiber surfaces [6] offers a more powerful interaction because it explicitly incorporates the topological evolution of the data samples in terms of the attribute values in the multivariate function. Nonetheless, we needed to investigate the radioactive deposition processes by understanding the mapping $\boldsymbol{R}^{2} \rightarrow \boldsymbol{R}^{3}$, while the fiber surface interface targeted $\boldsymbol{R}^{3} \rightarrow \boldsymbol{R}^{2}$. Thus, we focused on understanding the density distribution of the geospatial data samples in terms of the multiple attribute values, at the cost of missing the associated topological bifurcations in the range space. Figure 1 demonstrates a typical example of an interaction in which we tried to identify map regions that have specific patterns on the bivariate range spaces. Here, three pairs of arrows (red, green, and blue) represent such correspondences from the ranges to the map domain. A $32 \times 32$ grid was employed in each range window. Notice that the intensity at each grid is proportional to the number of connected components in the fiber at the corresponding function values, which means that bright colors indicate more densely projected data samples.

We also equipped our interface with the capability to track the correspondence from the data domain to the range by allowing users to drag the target region on the map. The orange arrows in Fig. 1 indicate such projections from a gray map area onto the three ranges. This helped us considerably when we already knew the specific radioactive deposition types for some of the regions. Furthermore, these bidirectional interactions compensated for the inability to analyze the details of the respective spaces when employing a unidirectional exploration only. This was especially helpful for visualization experts who did not have prior knowledge about projected patterns in the scatterplots according to the types of deposition processes and wanted to pursue such patterns by themselves. Note that we were able to selectively adjust the number of quantization levels in the ranges to a power of two, and a $64 \times 64$ grid was employed as the highest available resolution in our implementation.

\section{Preparation for the analysis}

In this section, we describe several preliminary setups for our visual analysis for categorizing radioactive deposition processes.

\subsection{Data acquisition}

In this study, the spatiotemporal distribution of the air dose rate around the FDNPP was obtained by airborne radiation monitoring, which has been conducted as a national project in Japan. Shortly after the FDNPP accident, a series of airborne radiation monitoring runs were organized by the project, and a human-crewed helicopter with measurement equipment was employed $[19,20]$. This successfully provided us with a detailed map of the surface distribution of radiocesium in the surrounding area. This airborne monitoring recorded ground surface gamma-ray radiation over a wide area, including mountainous regions that are difficult to approach from the ground. The crewed helicopter was also equipped with a global positioning system receiver, facilitating the precision of the helicopter's positioning when measuring the air dose rate. These measurements had a relatively fine spatial resolution (e.g., $250 \mathrm{~m}$ ). In the approximately $200 \mathrm{~km}$ radius around the FDNPP, flight spacing was $600,900,1800$, or $3000 \mathrm{~m}$ depending on the initial air dose rate. The flight speed and flight altitude were $150 \mathrm{~km} / \mathrm{h}$ and $300 \mathrm{~m}$, respectively. These measurement data also served as a basis for retrieving the corresponding topographic features of the sampling points, such as altitude, from the available digital elevation model data. Additional geospatial data samples, including segmented areas by land-use types (in 2009), were obtained from the data repository provided by the National Spatial Planning and Regional Policy Bureau, Ministry of Land, Infrastructure, Transport, and Tourism of Japan.

\subsection{Scientific explanation of deposition processes}

From a scientific point of view, we inferred the type of radioactive deposition process in each selected local area. Sanada et al. [23] successfully categorized representative local areas into dry and wet according to the type of radioactive deposition process. Their work presented profound understanding of the detailed deposition process in the respective areas by properly inferring the associated deposition models. They also took into account rainfall data, including the amount of precipitation after the accident and spatiotemporal behaviors of atmospheric radiocesium disparation, which were simulated based on the influence of wind direction and speed. This long-term statistical analysis helped us find meaningful patterns in the distribution of the air dose rate in terms of topographic features.

Sanada et al. [23] selected representative areas around the FDNPP and conducted environmental analyses of their radioactive deposition processes as exhibited in Fig. 4. The map (Fig. 4a) shows the two selected areas, and according to the legend (Fig. 4b), color is assigned in terms of the air dose rate and altitude. Through their detailed investigation, they categorized the two areas into dry and wet types (Fig. 4c). The dry deposition type corresponded to an area in which the radioactive material fell on terrain surfaces via turbulent flow and gravity precipitation, and thus the amount of the radioactive material was dependent on the 


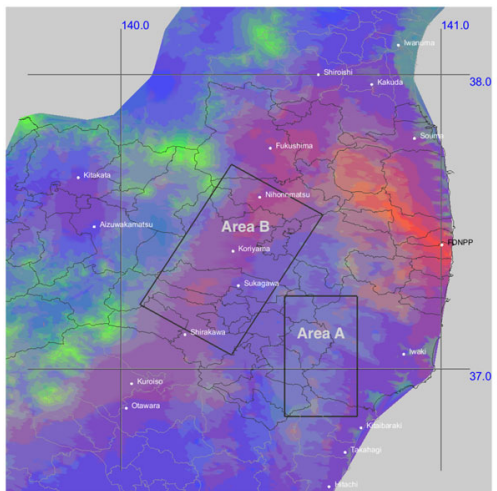

(a)

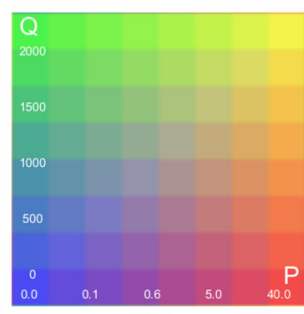

(b)

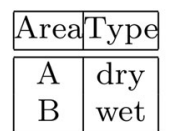

(c)
Fig. 4 Two representative local areas selected for the analysis of the radioactive deposition process [23]. a Areas A and B. b Color legend in terms of the air dose rate $(P)$ and altitude $(Q)$. $\mathbf{c}$ Inferred deposition types for the two areas. Air dose map measured on December 28, 2012. (cf. Sect. 5.1)

altitude and complexity of terrain surface shapes, which often made the corresponding distribution randomly scattered and the hotspots relatively isolated (Fig. 5a). In contrast, the wet deposition type occurred when the radioactive material was absorbed into rainfall and fell on an area, and thus, it is more likely to be uniformly distributed over the terrain surface regardless of the altitude (Fig. 5b).

Sanada et al. [23] created the histogram profiles of the air dose rate in terms of altitude. Analyzing these histogram profiles led us to the hypothesis that areas with a relatively high air dose rate should be categorized as dry at high altitudes or wet at low altitudes. This observation played a key role in identifying the type of radioactive deposition process using our system, which is detailed in the next section.

Evaluating the temporal change in the air dose rate is another important factor to develop an effective measure for protecting inhabitants from unwanted radiation exposure. For that purpose, it was important to discriminate radiation exposure caused by the FDNPP accident from that arising from natural radionuclide and cosmic rays. Sanada et al. [21] successfully established a method of calibrating the radiation level using the gamma energy spectra, regardless of the degree of background radiation levels. This method also allowed us to investigate how the radiocesium contamination spread deep into the soil, especially in wet deposition areas. According to [21], residential and agricultural areas are more likely to significantly suppress the associated air dose rate due to the effects of decontamination work and human activities, including agricultural work, construction work, and vehicle traffic. However, the decrease in the air dose rate in forest areas is expected to be constant and relatively small over time since the radiocesium compounds are absorbed deep in the soil [31]. We verified these expectations through visual analysis of geospatial multivariate data samples.

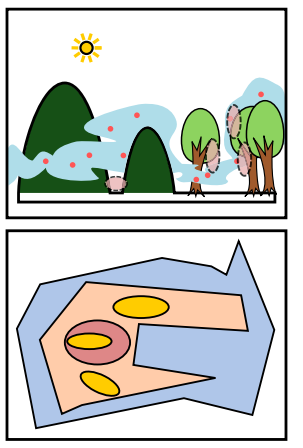

(a)

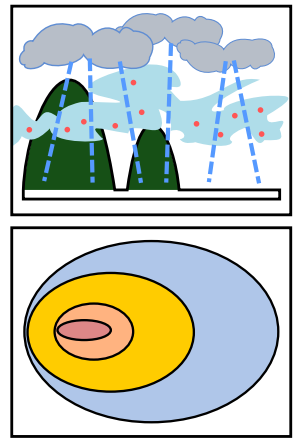

(b)
Fig. 5 Categorizing deposition processes into two types: a dry deposition type and $\mathbf{b}$ wet deposition type. In the bottom row, red indicates higher air dose rates, and blue indicates lower

\section{Experimental results}

In this section, we present the experimental results of our application study for the analysis of radioactive deposition processes around the FDNPP. We conducted our visual analysis with domain experts in the field (who are also authors of this manuscript). We implemented our prototype system on an Apple MacBook Pro laptop with Intel Core i7 with four cores $(2.3 \mathrm{GHz})$ and $32 \mathrm{~GB}$ of RAM. The source code was written in $\mathrm{C}++$ using the CGAL library for geometric computation, OpenGL for graphics, and GLUI for the interface. Our prototype system first approximated the geographical surface as a set of connected triangles for the given sample points over the area around the FDNPP. We then computed a set of three continuous scatterplots by projecting the triangles onto the 2D range space spanned by the corresponding pair of attribute values.

\subsection{Categorizing areas of dry and wet deposition types}

First, we projected terrain surfaces onto the 2D range spaces spanned by two of the three attributes, i.e., the air dose rate $(P)$, altitude from the ground $(Q)$, and distance from the FDNPP $(R)$ (Fig. 1). Our first challenge was to segment areas of dry and wet deposition types by drawing the patterns specific to each type over the set of 2D range windows. According to $[19,23]$, we observed common characteristics of each deposition type in the range spaces.

In areas of dry deposition type, turbulent airflows can carry radioactive materials, piling them high up in the mountains (see Fig. 5a). Additionally, the amount of material deposited was not significantly correlated with the distance from the nuclear power plant because it can travel for a relatively long distance through the air. The typical profiles of the data samples in the $P-Q$ and $P-R$ range spaces are illustrated in Fig. 6a. Alternatively, in the wet deposition processes, the 

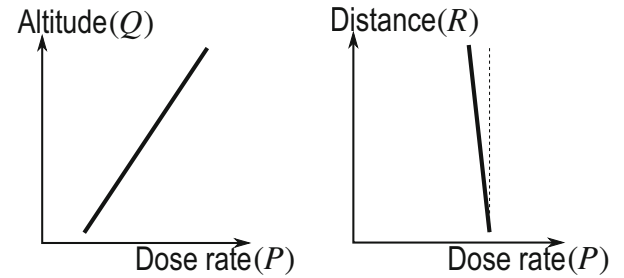

(a)
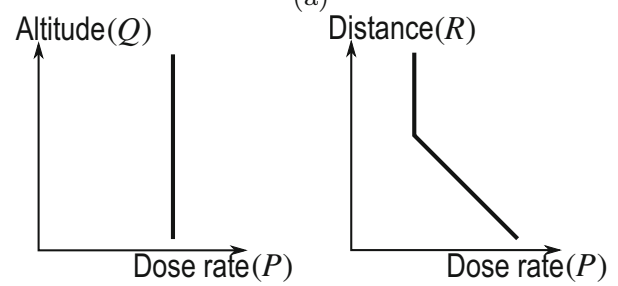

(b)

Fig. 6 Typical profiles in the projection of geospatial multivariate data in the range spaces in terms of the air dose rate, altitude from the ground, and distance from the FDNPP. a Dry deposition type and $\mathbf{b}$ wet deposition type

radioactive material falls the ground with rainfall, and thus the distribution is almost constant in terms of the elevation (see Fig. 5b). However, the possibility that the radioactive material will encounter rainfall while traveling through the air is roughly proportional to its closeness to the nuclear power plant. This implies that the data samples will have specific patterns in the range spaces as depicted in Fig. 6b for the wet deposition process areas.

This consideration allowed us to identify the specific deposition types in different areas by referring to the range spaces (Fig. 7). We employed geospatial samples from the air dose map measured on December 28, 2012. We limited the air dose rate range to $10^{-2}$ to $40.0 \mu \mathrm{Sv} / \mathrm{h}$ and then plotted the corresponding value in the logarithm scale. Each range window consisted of a $32 \times 32$ grid, and we drew specific patterns in the respective range windows to locate the typical areas of dry and wet deposition processes on the map around the FDNPP. We also interactively identified the corresponding areas on the map, with the horizontal and vertical axes corresponding to latitude and longitude scales, respectively.

Figure 7 a presents a case in which we found a potential area of dry deposition type on the map, which is represented by the colored pixels. Note that in this example, we first painted the pixels in the respective range windows by referring to Fig. 6a, and then, we identified the areas overlapping with the corresponding colored areas on the map. The result obtained in the map domain allowed us to confirm that Area $\mathrm{A}$ in Fig. 4a, which is enclosed by a rectangle in Fig. 7a, primarily consists of dry deposition areas, as already classified in Fig. 4c. In contrast, we located areas of wet deposition type by coloring the pixels that corresponded to the profiles in Fig. 6b. Area B of Fig. 4a, which is enclosed by a rectangle in Fig. 7b, is a wet deposition area as expected. Thus, we
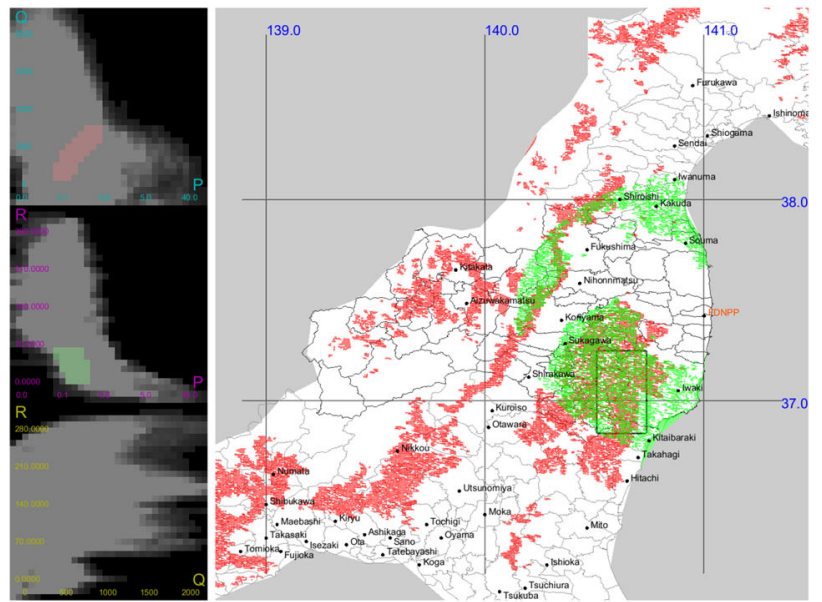

(a)
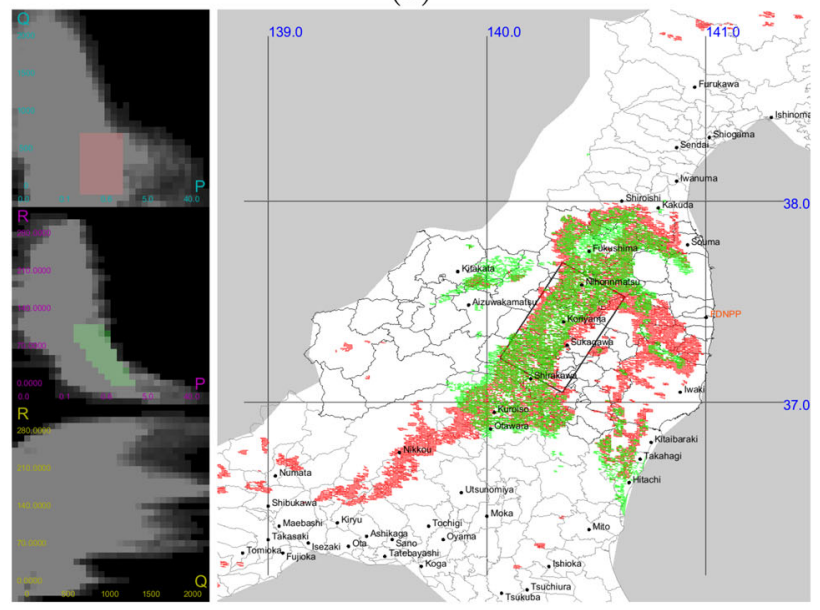

(b)
Fig. 7 Categorization of areas of dry and wet deposition types. Drawing specific patterns in the range windows allowed us to explore areas of (a) dry and (b) wet deposition processes within the map around the FDNPP

successfully categorized areas of specific deposition type by first drawing the corresponding typical patterns on the ranges based on the already acquired knowledge (Fig. 6), and then, we looked for the corresponding areas on the map domain.

\subsection{Analyzing temporal decreases in the air dose rate}

Our second challenge was to investigate the spatial distribution of radioactive contamination around the FDNPP and its temporal decreases according to the land-use type. We attempted to achieve this goal by focusing on the forest areas around the FDNPP, in which the radioactive material was expected to absorb deep into the soil due to rainfall (wet deposition processes). In this case study, we employed, as the third attribute value $R$, the exponent of the exponential fitting curve representing the attenuation in the air dose rate over 


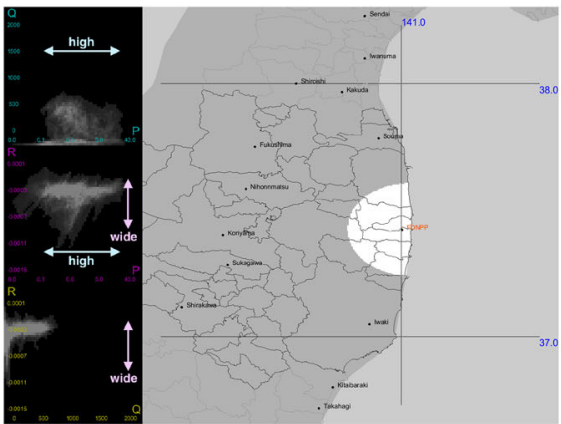

(a)
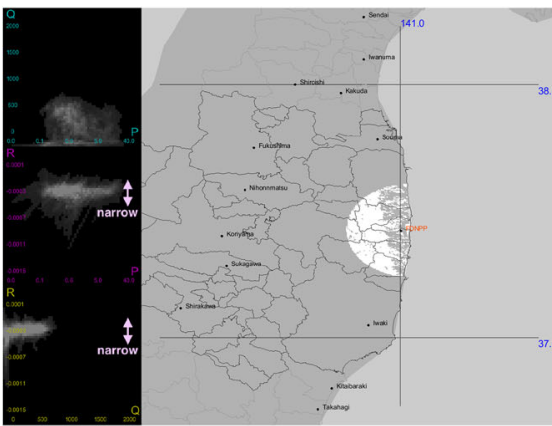

(b)

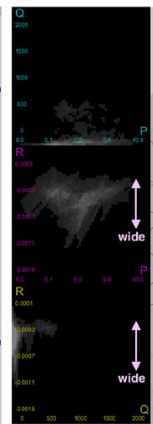

wide

Fig. 8 Analysis of the area within a $20 \mathrm{~km}$ radius of the FDNPP in terms of the air dose rate $(P)$, altitude from the ground $(Q)$, and the exponent of the exponential fitting curve for the temporal attenuation in the air dose rate $(R)$. a Entire area. b Forest area only. $\mathbf{c}$ Other areas, such as residential and agricultural areas

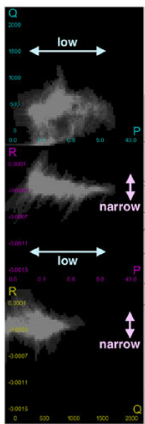

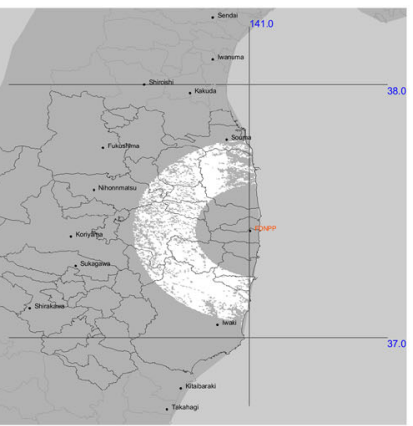

(a)
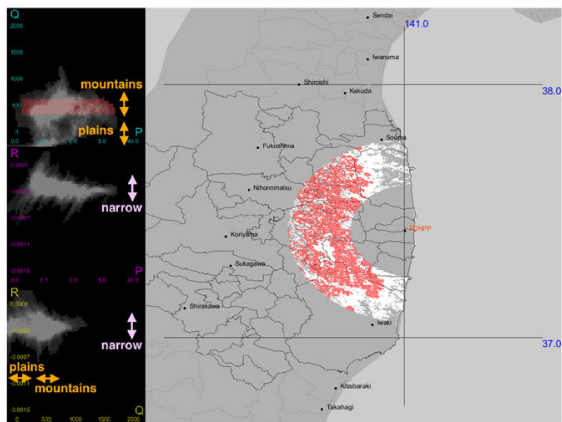

(b)
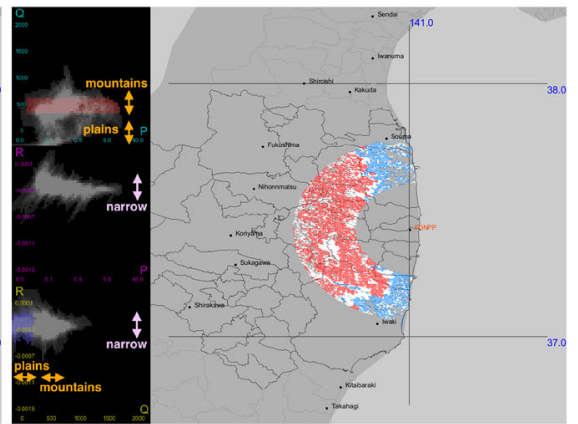

(c)

Fig. 9 Analysis of the area within a 20 to $40 \mathrm{~km}$ radius from the FDNPP. a Forest area only. b Areas with an altitude around $500 \mathrm{~m}$ are shown in red. c Areas with low altitudes (around the coastline) are shown in blue

time instead, along with the air dose rate $P$ and altitude from the ground $Q$. For this purpose, we approximated the exponential function by regression from nine temporal snapshots of the corresponding samples on the map obtained through monitoring campaigns conducted from 2012 to 2020 . The main flight condition was almost the same for every monitoring campaign. The attenuation exponent $R$ was calculated at each mesh $(250 \times 250 \mathrm{~m})$ using these data. Since we conducted this analysis to predict the future decrease in the radiation exposure caused by the FDNPP accident, we visualized the air dose rate at the map samples on the most recent measurement date, i.e., October 29, 2020.

In this experiment, we intended to conduct the following tasks through visual analysis of the spatial distribution of radioactive contamination.

(T1) Explain the attenuation in the air dose rate depending on the altitude and land-use type.

(T2) Infer the behavior of the radioactive plumes dissipating from the FDNPP after the accident.

We accomplished these tasks by partitioning the neighborhood of the FDNPP into two areas, one within a radius of 20 $\mathrm{km}$ from the FDNPP (Fig. 8) and the other within a radius of 20 to $40 \mathrm{~km}$ (Fig. 9). Note that in these two cases, we employed a $64 \times 64$ grid as the resolution of the range spaces onto which we projected a set of triangles constituting the map area.

First, we focused on the area within the $20 \mathrm{~km}$ radius around the FDNPP and explored how the attenuation in the air dose rate differed for each sample according to its landuse type. Figure 8 a shows the three projected images of the data samples contained in the area in terms of $P-Q, P-R$, and $Q-R$ ranges together with the visualization of the map domain around the FDNPP. You can easily see that the distribution of the attenuation exponents $R$ is relatively wide, as indicated by the light red arrows. We suspected that this was due to the human activities specific to areas of each land-use type, including agricultural work, construction work, vehicle traffic, and decontamination work, as described in Sect. 4.2. Thus, we restricted the data sample to the forest areas to exclude the residential and agricultural areas to examine the associated attenuation exponent ranges. Figure $8 \mathrm{~b}$ exhibits a case in which the distribution of the attenuation exponents in the forest area was concentrated in a narrow range, as represented by light red arrows. However, when projecting data 
samples of other land-use types (excluding the forest areas), we found a wide range of small attenuation exponents, as indicated by light red arrows in Fig. 8c. This observation led us to conclude that human activities, including decontamination work, resulted in a remarkable decrease in the air dose rate, especially in residential and agricultural areas. However, the radiocesium compounds were absorbed relatively deep into the soil in the forest areas due to the wet deposition process. This clearly justifies the difference in the distribution of $R$ raised in (T1).

The second case was the 20- to $40-\mathrm{km}$ area around the FDNPP (Fig. 9). Here, we limited our analysis to the forest areas only since the air dose rates are likely to reflect the damage directly caused by the accident in this case. Figure 9a presents the distribution of the three attribute values $(P, Q$, and $R$ ) in the target area around the FDNPP. By comparing the distributions of the air dose rate $P$ between Fig. $8 \mathrm{a}$ and Fig. 9a, as marked by light blue arrows, we learned that the air dose rate was high within the $20 \mathrm{~km}$ radius around the FDNPP but relatively low within the 20 to $40 \mathrm{~km}$ radius. Moreover, the $P-Q$ range window in Fig. $9 \mathrm{~b}$ suggests that the radioactive plumes were blocked by the Abukuma Mountains around an altitude of $500 \mathrm{~m}$, as painted in red. Notice that the Abukuma Mountain range runs north to south along the Pacific Ocean coast of Fukushima. This visual analysis successfully allowed us to infer how the radioactive plumes dissipated after the FDNPP accident (thus fulfilling T2). We were also interested in determining the difference in the attenuation of the air dose rate between the forest areas in the low plains and high mountains. The $P-R$ range map in Fig. 9c clearly reveals that the attenuation exponents in the forest areas were also within a narrow range, as indicated by light red arrows. Moreover, the $Q-R$ range map demonstrates that forest areas (indicated by orange arrows) in the mountains (painted in red) and in the plains (painted in blue) shared the same distribution of attenuation exponents. This allowed us to respond to (T1) by concluding that the attenuation exponents in the forest areas were almost constant regardless of their altitude from the ground. In other words, the air dose rate through wet deposition processes was not dependent on the altitude because rain caused the radioactive materials to fall suddenly (Fig. 6b).

\subsection{Discussion}

The hardware requirements for our approach were modest. Our analysis was indeed conducted on an ordinary laptop, the specifications of which can be found at the beginning of Sect. 5. Our implementation strategy allowed the system to compute an intermediate representation of the input geospatial data through a preprocess, which usually took approximately 200 seconds. In the meantime, it loaded input geospatial data along with the associated land-use types, then triangulated the map domain by referring to the sample points, and finally projected the triangles onto three continuous scatterplots. Once the system completed this preprocess, it could provide data at an almost interactive rate in the actual investigation of radioactive deposition processes and correspondence between specific areas in the map domain and their associated patterns projected on the scatterplots. The highest resolution for the continuous scatterplot in our system was $64 \times 64$ since it provided sufficiently fine details of areas on the map domain. Other lower-resolution grids were obtained by aggregating pixels in this original highest resolution in our level-of-detail representation and thus were instantly computed. Further accelerating the preprocess might require parallel computation, which we will explore in a future work.

The proposed interaction scheme also offered non-experts in this application domain an opportunity to explore the meaningful patterns in the geospatial multivariate data. This was achieved through bidirectional exploration between the map domain and their projections onto the continuous scatterplots. As described earlier, the unidirectional interaction helped us identify areas of specific deposition types on the map domain by drawing typical patterns on the scatterplots in terms of attribute values. This operation was beneficial especially when we had prior knowledge about radioactive deposition processes. At the same time, we were also able to explore such important patterns by projecting areas of interest onto the scatterplots with the reverse interaction. This trialand-error scheme compensated for the inability to directly discover meaningful areas from a scientific perspective.

This work is also practically advantageous since it allowed domain experts to interactively confirm their hypotheses on the temporal change in the air dose rate regarding deposition types and land-use patterns. This advantage could also allow us to predict possible countermeasures to alleviate the associated soil pollution problems. A straightforward task might be to identify radioactive deposition types in uninvestigated areas by referring to the scientific insights obtained from our prior visual analysis. We could also design effective decontamination work according to the radioactive deposition types, temporal changes in the dose rate, and the associated land-use types. For example, we expect that the decontamination work greatly reduces the unwanted radiation levels if the target area is of a dry deposition type. Conversely, if the area is a wet deposition area, especially in the forests, the radioactive material is likely to soak deep into the soil. In this case, further research is needed to enhance the effects of the decontamination work. This may help us schedule the overall decontamination work in the contaminated area around FDNPP to maximize the decrease in the dose rate with limited time and human resources.

We believe that the proposed approach may be applied to the visual analysis of soil pollution caused by other par- 
ticulate matter, including hazardous chemicals emitted from factories and garbage collection sites. In this case, we would need to discover attribute values that influence the spatial distribution of such particulate matter and their patterns associated with soil pollution. One potential application is to understand more complicated air pollution caused by suspended particulate matter, including PM2.5 and Asian Dust. Proper models may be required to understand the deposition of such particulate matter on human respiratory tracts.

One potential advancement in the relevant fields would be the ability to simultaneously incorporate four or more attribute values in the analysis of the multivariate data. Solving this problem may require integration with visualization techniques for multivariate data analysis, such as scatterplot matrices and parallel coordinate plots. Being able to automatically find a correlated set of attribute values based on their geographical positions would greatly facilitate interesting scientific discoveries in the multivariate data. A deeper understanding of radioactive deposition processes and their associated environmental influences is also needed. The actual processes of radioactive deposition are sometimes unexpectedly complicated due to the uncertain behavior of wind and rain immediately after a nuclear accident. This implies the need for a more sophisticated, level-of-detail control over the analysis of dense samples in local areas. Introducing more relevant attribute values into our visual analysis could enhance our ability to devise more effective decontamination plans.

\section{Conclusion}

In this paper, we presented an application study for analyzing radioactive deposition processes in the area around the FDNPP accident site. We visually investigated the correspondence between the spatial position and air dose rate together with additional topographic attributes. We implemented an interactive system for visualizing the continuous scatterplots of the multivariate function defined over the area, and we identified areas of specific deposition processes by exploring meaningful patterns in the range spaces. This was achieved by properly modeling radioactive deposition processes and collecting knowledge through statistical analyses of the relevant data obtained by airborne radiation monitoring. We conducted several experiments to demonstrate that our approach can provide useful insight into the analysis of radioactive deposition processes and their impact on the temporal change in the air dose rate.

Acknowledgements This work was partially supported by JSPS KAKENHI (Grant Numbers 19H04120, 16H02825, and 20K19809) and is partially based on discussions at the 2021 IMI Joint Use Research Program Workshop (I) "Fiber Topology Meets Applications 2."
Open Access This article is licensed under a Creative Commons Attribution 4.0 International License, which permits use, sharing, adaptation, distribution and reproduction in any medium or format, as long as you give appropriate credit to the original author(s) and the source, provide a link to the Creative Commons licence, and indicate if changes were made. The images or other third party material in this article are included in the article's Creative Commons licence, unless indicated otherwise in a credit line to the material. If material is not included in the article's Creative Commons licence and your intended use is not permitted by statutory regulation or exceeds the permitted use, you will need to obtain permission directly from the copyright holder. To view a copy of this licence, visit http://creativecomm ons.org/licenses/by/4.0/.

\section{References}

1. Andrienko, G., et al.: Space-in-time and time-in-space selforganizing maps for exploring spatiotemporal patterns. Comput. Graph. Forum 29(3), 913-922 (2010). https://doi.org/10.1111/j. 1467-8659.2009.01664.x

2. Bachthaler, S., Weiskopf, D.: Continuous scatterplots. IEEE Trans. Vis. Comput. Graph. 14(6), 1428-1435 (2008). https://doi.org/10. 1109/TVCG.2008.119

3. Beecham, R., et al.: Faceted views of varying emphasis (FaVVEs): a framework for visualising multi-perspective small multiples. Comput. Graph. Forum 35(3), 241-249 (2016). https://doi.org/10. $1111 /$ cgf. 12900

4. Butkiewicz, T., Dou, W., Wartell, Z., Ribarsky, W., Chang, R.: Multi-focused geospatial analysis using probes. IEEE Trans. Vis. Comput. Graph. 14(6), 1165-1172 (2008). https://doi.org/10. 1109/TVCG.2008.149

5. Carr, H., Duke, D.: Joint contour nets. IEEE Trans. Vis. Comput. Graph. 20(8), 1100-1113 (2014). https://doi.org/10.1109/TVCG. 2013.269

6. Carr, H., Geng, Z., Tierny, J., Chattopadhyay, A., Knoll, A.: Fiber surfaces: Generalizing isosurfaces to bivariate data. Comput. Graph. Forum 34(3), 241-250 (2015). https://doi.org/10.1111/cgf. 12636

7. Chattopadhyay, A., Carr, H., Duke, D., Geng, Z., Saeki, O.: Multivariate topology simplification. Comput. Geom. Theory and Appl. 58(C), 1-24 (2016). https://doi.org/10.1016/j.comgeo.2016. 05.006

8. Duke, D., et al.: Visualizing nuclear scission through a multifield extension of topological analysis. IEEE Trans. Vis. Comput. Graph. 18(12), 2033-2040 (2012). https://doi.org/10.1109/TVCG.2012. 287

9. Edelsbrunner, H., Harer, J.: Jacobi sets of multiple Morse functions. In: Foundations of Computational Mathematics, pp. 37-57 (2004). https://doi.org/10.1017/CBO9781139106962.003

10. Edelsbrunner, H., Harer, J., Patel, A.K.: Reeb spaces of piecewise linear mappings. In: Proceedings of the Twenty-Fourth Annual Symposium on Computational Geometry, pp. 242-250 (2008). https://doi.org/10.1145/1377676.1377720

11. Klacansky, P., Tierny, J., Carr, H., Geng, Z.: Fast and exact fiber surfaces for tetrahedral meshes. IEEE Trans. Vis. Comput. Graph. 23(7), 1782-1795 (2017). https://doi.org/10.1109/TVCG. 2016.2570215

12. Krüger, R., Thom, D., Wörner, M., Bosch, H., Ertl, T.: Trajectorylenses - A set-based filtering and exploration technique for long-term trajectory data. Comput. Graph. Forum 32(3), 451-460 (2013). https://doi.org/10.1111/cgf.12132

13. Lehmann, D.J., Theisel, H.: Discontinuities in continuous scatter plots. IEEE Trans. Vis. Comput. Graph. 16(6), 1291-1300 (2010). https://doi.org/10.1109/TVCG.2010.146 
14. Liu, H., et al.: Visual analysis of route diversity. Proc. IEEE VAST 2011, 171-180 (2011). https://doi.org/10.1109/VAST.2011. 6102455

15. Maciejewski, R., et al.: A visual analytics approach to understanding spatiotemporal hotspots. IEEE Trans. Vis. Comput. Graph. 16(2), 205-220 (2010). https://doi.org/10.1109/TVCG.2009.100

16. Meulemans, W., Dykes, J., Slingsby, A., Turkay, C., Wood, J.: Small multiples with gaps. IEEE Trans. Vis. Comput. Graph. 23(1), 381-390 (2017). https://doi.org/10.1109/TVCG.2016.2598542

17. Saeki, O.: Topology of singular fibers of differentiable maps, vol. 1854. Springer (2004)

18. Sakurai, D., et al.: Interactive visualization for singular fibers of functions $f: \boldsymbol{R}^{3} \rightarrow \boldsymbol{R}^{2}$. IEEE Trans. Vis. Comput. Graph. 22(1), 945-954 (2016). https://doi.org/10.1109/TVCG.2015.2467433

19. Sanada, Y., Ishizaki, A., Nishizawa, Y., Urabe, Y.: Airborne radiation monitoring using a manned helicopter (in Japanese). Anal. Chem. 66(3), 149-162 (2017). https://doi.org/10.2116/ bunsekikagaku.66.149

20. Sanada, Y., Sugita, T., Nishizawa, Y., Torii, A.: The airborne radiation monitoring in Japan after the Fukushima Daiichi nuclear power plant accident. Progress Nucl. Sci. Technol. 4, 76-80 (2014). https://doi.org/10.15669/pnst.4.76

21. Sanada, Y., Urabe, Y., Sasaki, M., Ochi, K., Torii, T.: Evaluation of ecological half-life of dose rate based on airborne radiation monitoring following the Fukushima Dai-ichi nuclear power plant accident. J. Environ. Radioact. 210,(2019). https://doi.org/ 10.1016/j.jenvrad.2018.09.014

22. Sanada, Y., et al.: Radiation monitoring using an unmanned helicopter in the evacuation zone around the Fukushima Daiichi nuclear power plant. Explor. Geophys. 45(1), 3-7 (2014). https://doi.org/ 10.1071/EG13004

23. Sanada, Y., et al.: Altitudinal characteristics of atmospheric deposition of aerosols in mountainous regions: Lessons from the Fukushima Daiichi nuclear power station accident. Sci. Total Environ. 618, 881-890 (2018). https://doi.org/10.1016/j.scitotenv. 2017.08.246

24. Sopan, A., et al.: Community health map: A geospatial and multivariate data visualization tool for public health datasets. Gov. Inf. Q. 29(2), 223-234 (2012). https://doi.org/10.1016/j.giq.2011.10. 002

25. Tatu, A., et al.: Subspace search and visualization to make sense of alternative clusterings in high-dimensional data. Proc. IEEE VAST 2012, 63-72 (2012). https://doi.org/10.1109/VAST.2012.6400488

26. Tierny, J., Carr, H.: Jacobi fiber surfaces for bivariate Reeb space computation. IEEE Trans. Vis. Comput. Graph. 23(1), 960-969 (2017). https://doi.org/10.1109/TVCG.2016.2599017

27. Tominski, C., Gladisch, S., Kister, U., Dachselt, R., Schumann, H.: Interactive lenses for visualization: An extended survey. Comput. Graph. Forum 36(6), 173-200 (2017). https://doi.org/10.1111/cgf. 12871

28. Turkay, C., Lundervold, A., Lundervold, A.J., Hauser, H.: Representative factor generation for the interactive visual analysis of high-dimensional data. IEEE Trans. Vis. Comput. Graph. 18(12), 2621-2630 (2012). https://doi.org/10.1109/TVCG.2012.256

29. Turkay, C., Slingsby, A., Hauser, H., Wood, J., Dykes, J.: Attribute signatures: Dynamic visual summaries for analyzing multivariate geographical data. IEEE Trans. Vis. Comput. Graph. 20(12), 20332042 (2014). https://doi.org/10.1109/TVCG.2014.2346265

30. Watanabe, K., Wu, H.Y., Niibe, Y., Takahashi, S., Fujishiro, I.: Biclustering multivariate data for correlated subspace mining. Proc. IEEE PacificVis 2015, 287-294 (2015). https://doi.org/10.1109/ PACIFICVIS.2015.7156389

31. Yasuda, Y.: Air dose rate in forests and its temporal changes for the past five years. Water Sci. 61(5), 102-130 (2017). https://doi. org/10.20820/suirikagaku.61.5_102. (in Japanese)

32. Yates, A., et al.: Visualizing multidimensional data with glyph SPLOMs. Comput. Graph. Forum 33(3), 301-310 (2014). https:// doi.org/10.1111/cgf.12386

33. Yuan, X., Ren, D., Wang, Z., Guo, C.: Dimension projection matrix/tree: Interactive subspace visual exploration and analysis of high dimensional data. IEEE Trans. Vis. Comput. Graph. 19(12), 2625-2633 (2013). https://doi.org/10.1109/TVCG.2013.150

Publisher's Note Springer Nature remains neutral with regard to jurisdictional claims in published maps and institutional affiliations.

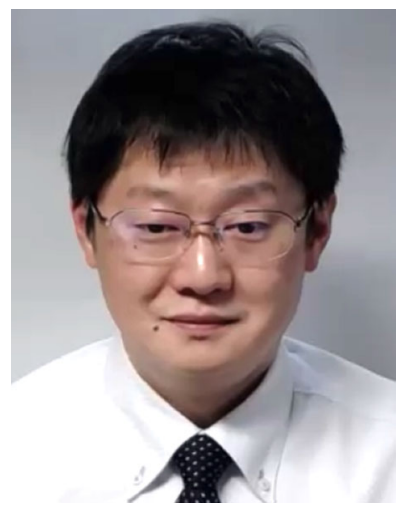

Shigeo Takahashi is currently a professor at the School of Computer Science and Engineering at the University of Aizu, Japan. He received his B.S., M.S., and Ph.D. in computer science from the University of Tokyo in 1992, 1994, and 1997, respectively. His research interests include scientific and information visualization, visual perception modeling, geographic/cartographic visualization, and geometric modeling. $\mathrm{He}$ is currently serving as an associate editor of the journal Computational Visual Media. He received the Most Cited Paper Award for Graphical Models (2004-2006) in 2007 from Elsevier.

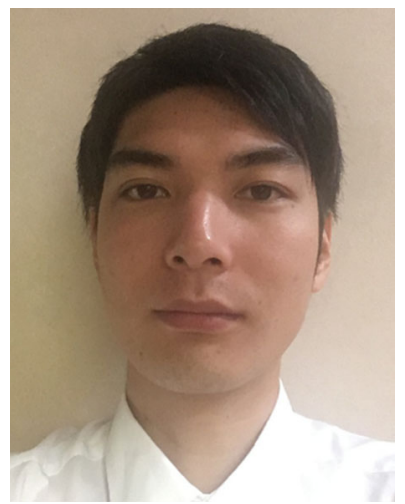

Daisuke Sakurai is an associate professor at Pan-Omics Data Drive Innovation Research Center at the Research Institute for Information Technology of Kyushu University, Japan. He received his B.S. in science from Hokkaido University in 2010 and M.S. and Ph.D. in science from the University of Tokyo in 2012 and 2015, respectively. His research interests include scientific visualization, topological data analysis, and multi-objective optimization. He studies techni ques for analyzing visual data in collaboration with application fields, especially meteorology.

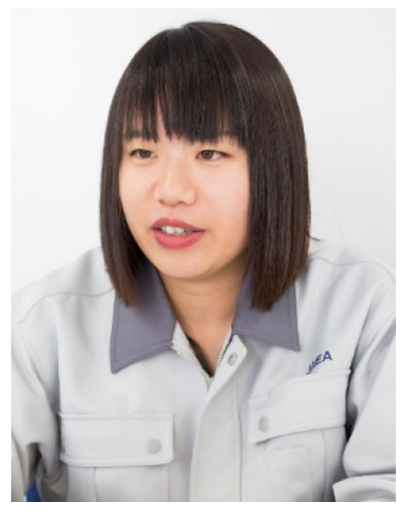

surement using machine learning.

Miyuki Sasaki is a researcher at the Sector of Fukushima Research and Development of the Japan Atomic Energy Agency. Her specialty is radiation measurement. She received her B.S. and M.S. in analytical chemistry from Fukushima University in 2013 and 2015 , respectively. She received her $\mathrm{Ph} . \mathrm{D}$. in nuclear engineering from Nagoya University in 2021. Her interest is computational radiation science. She is researching and developing a new analytical method for remote radiation mea- 


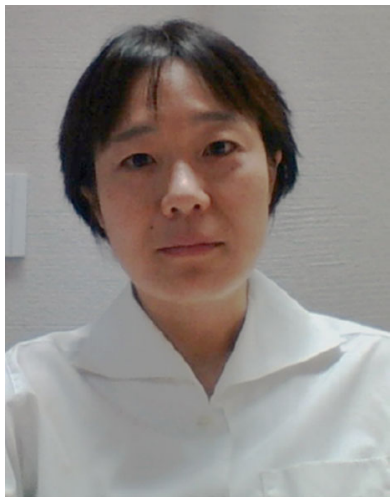

Hiroko N. Miyamura is currently an engineer at the Center for Computational Science and eSystems and the Nuclear Human Resource Development Center of the Japan Atomic Energy Agency. She received her B.S. and M.S. in information sciences and her $\mathrm{Ph} . \mathrm{D}$. in computer science from Ochanomizu University, in 1999 , 2001, and 2004, respectively. Her research interests are scientific visualization, information visualization, and computer graphics.

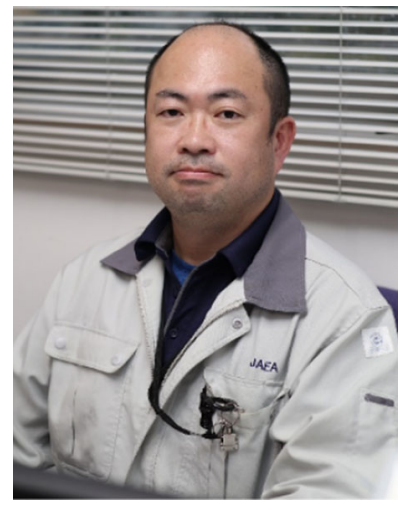

Yukihisa Sanada is a principal engineer at the Sector of Fukushima Research and Development of the Japan Atomic Energy Agency. His specialty is radiation measurement. $\mathrm{He}$ received his B.S. and M.S. in environmental science from Tokyo University of Agriculture and Technology in 1997 and 1999, respectively. He received his Ph.D. in analytical chemistry from Niigata University in 2006. He is conducting research and development on an advanced radiation detector. Since the Fukushima Daiichi NPP accident in 2011, he has been performing remote radiation surveys such as aerial radiation monitoring using UAV. 